Insgesamt fanden sich bei den zehn Mädchen 29 HPV-Infektionen mit onkogenen (HPV 66, 39, 51, 16) sowie nicht-onkogenen (HPV 42, 62, 6, 11) HPV-Typen. Bei nicht-koitalen Sexualkontakten gaben sie Zungenküssse (81,8\%), Berührung der Genitalien des Partners oder Berührung der eigenen Genitalien durch den Partner (54,6\%) vor dem ersten Koitus an. Ferner wurde oro-genitaler Kontakt $(18,2 \%)$ sowie rezeptiver oro-genitaler Kontakt $(22,7 \%)$ genannt.
Shew ML et al. Frequent detection of vaginal human papillomavirus prior to first sexual intercourse during longitudinal observation. J Infect Dis 2012 Dec 13; (Epub ahead of print)

Kommentar: Auf nichtkoitale Sexualkontakte wurde bei der Diskussion über das richtige Startalter der HPV-Impfung bisher nur wenig geachtet. Diese Longitudinalstudie belegt jedoch, dass nahezu die Hälfte der adoleszenten Mädchen schon vor dem ersten vaginalen Koitus

\title{
Kindliche Halo-Nävi und das Risiko für Vitiligo
}

Der Halo-Nävus, für den auch synonym nach seinem Erstbeschreiber der Begriff Sutton-Nävus verwendet wird, ist eine gutartige, erworbene Depigmentierung um einen Nävuszellnävus. Er tritt häufig zusammen mit Vitiligo auf.

$\mathrm{H}$ alo-Nävi manifestieren sich überwiegend am Stamm und können singulär oder multipel auftreten. Klinisch können Halo-Nävi in vier Phasen vorkommen (Tabelle 1).

Das Auftreten von Halo-Nävi bei Patienten mit Vitiligo ist ein klinisch immer wieder beobachtetes Phänomen. Diese Zusammenhänge haben auch gemeinsame pathogenetische Wurzeln, da immunhistopathologische Untersuchungen zytotoxische T-Lymphozyten, sowohl in läsionaler Haut von Patienten mit nichtsegmentaler Vitiligo als auch in Halo-Nä-

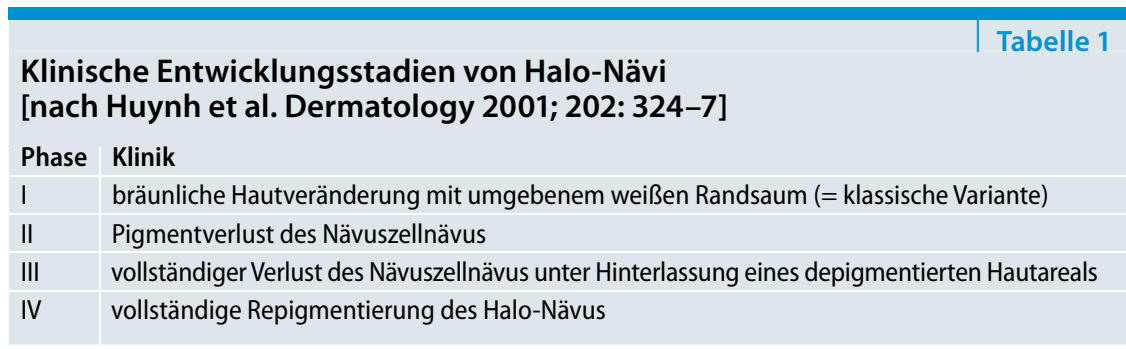



Abb. 1: Singulärer Halo-Nävus: kein erhöhtes Risiko für Vitiligo und Autoimmunerkrankungen

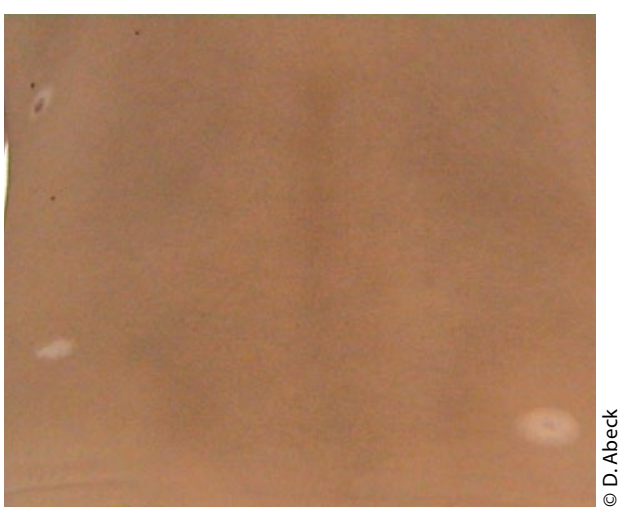

Abb. 2: Multiple Halo-Nävi: erhöhtes Risiko für Vitiligo und Autoimmunerkrankungen durch diverse nicht-koitale Sexualpraktiken mit HPV infiziert sein können. Auch in früheren Untersuchungen wurde die Rolle von Fingern bei der Übertragung von HPV bestätigt. Die Studie verdeutlicht eindringlich die Bedeutung einer frühzeitigen HPV-Impfung. Beide HPV-Impfstoffe sind ab dem 9. Lebensjahr zugelassen, werden aber wegen der geltenden STIKO-Empfehlung in der Regel erst ab dem 12. Lebensjahr angewendet.

Prof. Dr. Tino F. Schwarz

vi nachweisen konnten. In der vorliegenden retrospektiven Untersuchung bei Kindern wurde einem möglichen Zusammenhang zwischen Halo-Nävi und Vitiligo nachgegangen. Gruppe I umfasste 40 Kinder mit Halo-Nävi, Gruppe II 173 Kinder mit nicht-segmentaler Vitiligo und Gruppe III 78 Kinder mit nicht-segmentaler Vitiligo und Halo-Nävi.

Beim Vergleich der Kinder von Gruppe I mit Kindern der Gruppe II bestanden bei Kindern mit ausschließlich Halo-Nävi weniger Autoimmunerkrankungen ( $\mathrm{p}$ $<0,001$ ), häufiger eine negative FamilienAnamnese für Vitiligo $(\mathrm{p}<0,013)$ und seltener ein Köbner-Phänomen ( $<<0,001)$. Beim Vergleich von Kindern der Gruppe I und Gruppe III bei Berücksichtigung nur der Kinder mit multiplen Halo-Nävi konnte ein Zusammenhang mit dem häufigeren Auftreten von Vitiligo festgestellt $(\mathrm{p}<0,006)$ werden.

Patriz A et al. Association of halo nevus/i and vitiligo in childhood: a retrospective observational study. J Eur Acad Dermatol Venereol 2013; 27: e148-52

Kommentar: Die Untersuchungen legen den Schluss nahe, dass zwei verschiedene klinische Präsentationsformen von HaloNävi im Kindesalter existieren: zum einen ein absolut benignes, zeitlich begrenztes Phänomen ohne Korrelation mit assoziierten Autoimmunerkrankungen (Abb. 1) wobei dieser Typ überwiegend durch einzelne und spontan regressive Halo-Nävi gekennzeichnet ist. Dagegen besteht beim Auftreten von multiplen Halo-Nävi ein erhöhtes Risiko für Vitiligo und assoziierte Autoimmunerkrankungen (Abb. 2), insbesondere die der Schilddrüse.

Prof. Dr. Dietrich Abeck 